\title{
Comparación del nivel de nitrógeno ureico sanguíneo entre alpacas y llamas destetadas mantenidas en pastos cultivados
}

\author{
Comparison of blood urea nitrogen level between weaned alpacas and llamas \\ grazing cultivated pastures
}

\author{
Mery Vivar H. ${ }^{1}$. Juan Olazábal L. ${ }^{1,2}$, Felipe San Martín H. ${ }^{1}$
}

\section{Resumen}

\begin{abstract}
El presente estudio tuvo como objetivo comparar el nivel del nitrógeno ureico sanguíneo (NUS) entre alpacas y llamas destetadas, mantenidas en pastos cultivados. Se utilizaron 63 alpacas y 64 llamas, tanto hembras como machos, mantenidas por 21 días luego del destete sobre pasturas cultivadas (asociación de rye grass italiano [Lolium multiflorum] y trébol blanco [Trifolium repens]). La toma de la muestra de sangre se hizo el día 21 antes que salgan a pastorear. Se evaluó la composición química del alimento ofrecido, el NUS y el índice de selectividad (IS). La determinación del NUS se hizo por espectrofotometría UV visible con un kit comercial. El IS se determinó entre la composición botánica del potrero y la composición botánica del consumo. Los datos fueron analizados a través de la prueba de t Student de dos muestras con varianzas iguales. La composición química del pasto cultivado ofrecido para el caso de materia seca, proteína cruda, fibra cruda, extracto etéreo y ceniza fue de 18.09 , 16.30, 19.20, 1.43 y $6.60 \%$, respectivamente. Los valores de NUS en alpacas y llamas fueron de $19.04 \pm 3.24 \mathrm{mg} / \mathrm{dl}$ (12.13 $26.36 \mathrm{mg} / \mathrm{dl})$ y $19.05 \pm 3.93 \mathrm{mg} / \mathrm{dl}(8.80-25.69 \mathrm{mg} / \mathrm{dl})$, respectivamente, sin diferencia significativa entre especies. El IS fue neutro, no encontrándose selectividad en ambas especies. Se concluye que el NUS es similar entre alpacas y llamas destetadas mantenidas en pasturas cultivas y que no presentaron selectividad para el consumo.
\end{abstract}

Palabras clave: nitrógeno ureico sanguíneo; pastos cultivados; alpacas; llamas

\section{AbSTRACT}

The aim of this study was to compare the level of blood urea nitrogen (BUN) between weaned alpacas and llamas grazing cultivated pastures. It was used 63 alpacas and 64 llamas, both females and males, maintained for 21 days after weaning on cultivated pastures

\footnotetext{
${ }^{1}$ Laboratorio de Bioquímica, Nutrición y Alimentación Animal, Facultad de Medicina Veterinaria, Universidad Nacional Mayor de San Marcos, Lima, Perú

${ }^{2}$ E-mail: jolazaball@unmsm.edu.pe
}

Recibido: 8 de junio de 2018

Aceptado para publicación: 16 de noviembre de 2018 
(association of Italian rye grass [Lolium multiflorum] and white clover [Trifolium repens]). The blood sample was taken on day 21 of the study before animals went for grazing. The chemical composition of the offered feed, BUN and the selectivity index (SI) were evaluated. The BUN determination was done by visible UV spectrophotometry with a commercial kit. The IS was determined between the botanical composition of the pasture and the botanical composition of feed consumed. The data were analyzed through the Student $t$ test of two samples with equal variances. The chemical composition of the cultivated grass offered for dry matter, crude protein, crude fiber, ethereal extract and ash was $18.09,16.30,19.20,1.43$ and $6.60 \%$, respectively. BUN values in alpacas and llamas were $19.04 \pm 3.24 \mathrm{mg} / \mathrm{dl}(12.13-26.36 \mathrm{mg} / \mathrm{dl})$ and $19.05 \pm 3.93 \mathrm{mg} / \mathrm{dl}(8.80-25.69 \mathrm{mg} / \mathrm{dl})$, respectively, without significant difference between species. The IS was neutral, and no selectivity was found in both species. It is concluded that BUN is similar between weaned alpacas and llamas grazing cultivated pastures and they did not show selectivity for consumption.

Key words: blood urea nitrogen; cultivated pastures; alpacas; llamas

\section{INTRODUCCIÓN}

Los camélidos sudamericanos (CSA) se encuentran mejor adaptados a las condiciones ambientales adversas de las regiones andinas, como la altura, la radiación solar, el frío y al consumo de forrajes de baja calidad con relación a los bovinos, ovinos y caprinos, criados bajo las mismas condiciones (Van Saun, 2006).

La eficiencia en el uso de los alimentos pobres por los CSA está relacionado con sus características anatómicas y fisiológicas. Tienen tres compartimentos estomacales, presentan sacos glandulares, mayor tiempo de retención del alimento en el tracto digestivo, mayor frecuencia de contracciones en el estómago, mayor flujo salival, mayor eficiencia en el reciclaje y utilización de la urea corporal y, por consiguiente, se facilita más nitrógeno disponible para la síntesis microbiana, mejorando la digestibilidad y permitiendo la producción de energía y proteína para el mantenimiento y producción de los animales (San Martín, 1996a; Van Saun, 2006).

El nitrógeno ureico sanguíneo (NUS) es la cantidad de nitrógeno circulando en el torrente sanguíneo en forma de urea y está for- mado a partir del exceso de amoniaco producido en el rumen, que al ser absorbido en la pared epitelial del rumen es transportado al hígado donde es transformado en urea. Esta puede reingresar al rumen mediante su pared ruminal o por la saliva (reciclaje de urea) o ser transportado al riñón para excretarse en la orina (Van Saun, 2006). La concentración de NUS en los rumiantes indica el nivel de proteína de la dieta (Arias y Nesti de Alonso, 1999; Van Saun, 2006).

Uno de los periodos nutricionales críticos en la crianza de los CSA es el destete, el cual se realiza entre los meses de setiembre y octubre, meses que coinciden con una baja en la disponibilidad y calidad del forraje de los pastizales altoandinos. Para superar esta etapa de mayor requerimiento nutritivo y poder alcanzar un peso adecuado para el empadre se han diseñado varias alternativas alimenticias, siendo una de ellas el uso de pastos cultivados (San Martín, 1996b).

Los pastos cultivados se caracterizan por ser una fuente de alta calidad nutritiva (carbohidratos y proteína) que pueden proporcionar a los animales destetados los nutrientes necesarios para cumplir con sus funciones de crecimiento y alcanzar un peso vivo adecuado para el empadre, que en el 
caso de alpacas es 33-36 kg y en llamas de 50-55 kg (García et al., 1999); sin embargo, se sabe que las llamas están mejor adaptadas a consumir pastos de menor calidad que las alpacas. Se conoce que la llama tiene una mayor preferencia por gramíneas altas, mientras que la alpaca tiene una alta selectividad por plantas herbáceas (San Martín, 1996a).

Además, se sabe que el NUS, tanto en alpacas como en llamas, es elevado en comparación con los rumiantes, pero se desconoce la respuesta del NUS en alpacas y llamas destetadas alimentadas con pasturas cultivadas de alta calidad proteica. Por eso, el presente estudio tuvo como objetivo comparar el nivel de nitrógeno ureico sanguíneo entre alpacas y llamas destetadas mantenidas en pastos cultivados, planteando como hipótesis que los niveles de NUS son mayores en alpacas destetadas que en llamas destetadas mantenidas en pastos cultivados por la alta selectividad de las alpacas por plantas herbáceas ricas en proteína.

\section{Materiales y Métodos}

\section{Lugar de Ejecución}

La parte experimental del trabajo se realizó en la Estación IVITA Maranganí, ubicada en el distrito de Maranganí, provincia de Canchis, región Cusco (Perú), a una altura de $3700 \mathrm{msnm}$, durante los meses de setiembre y octubre de 2017. El análisis de las muestras se realizó en el Laboratorio de Bioquímica, Nutrición y Alimentación Animal (LBNAA) de la Facultad de Medicina Veterinaria (FMV) de la Universidad Nacional Mayor de San Marcos (UNMSM), Lima.

\section{Animales}

Se utilizaron 127 animales (63 alpacas y 64 llamas), tanto tuis hembras como tuis machos destetados (4-7 meses de edad), aparentemente sanos. La alimentación fue con pastos cultivados asociación de rye grass italiano (Lolium multiflorum) y trébol blanco
(Trifolium repens), en una proporción de 82.1 y $17.9 \%$ respectivamente. Durante el estudio se siguieron las condiciones de manejo del pastoreo, riego cada 7 días, descanso de $50 \mathrm{~d}$ y abonamiento con heces de cuyes, que es el manejo que realiza la estación experimental para pastos cultivados con animales destetados.

Día 1: Los animales fueron pesados y destetados. Se les llevó a la zona de pastoreo de pastos cultivados. Se formó un potrero de $350 \mathrm{~m}^{2}(7 \times 50 \mathrm{~m})$ con un cerco eléctrico de tres hilos, para ambas especies. El horario de pastoreo fue entre las 08:00 y las 17:00, sin recibir suplemento alguno durante el encierro nocturno. Nuevas áreas de pastoreo con similares medidas fueron establecidas diariamente.

Día 20: Toma de muestras para el análisis de la composición química del pasto cultivado ofrecido, para la composición botánica del potrero y para la composición botánica del consumo de los animales.

Día 21: Toma de muestra de sangre a las 06:00, en ayunas y previo a la salida al pastoreo.

\section{Composición Botánica y Análisis Quími- co Proximal}

El muestreo de la pastura se realizó el día 20 del experimento, antes que los animales ingresen al potrero. Se hizo un muestreo estratificado del potrero (según la altura del crecimiento), tomando cinco muestras representativas de $1 \mathrm{~m}^{2}$ cada, determinadas por la proporción de altura de la pastura, que luego fueron homogenizadas y pesadas en base fresca. Estas muestras se utilizaron para determinar la composición botánica del potrero y para realizar el análisis químico proximal de las plantas. Se tomaron submuestras y se hizo la separación manual de gramíneas y leguminosas y se pesaron por separado para determinar la proporción de especies de gramíneas y leguminosas presentes en el potrero. El análisis proximal de humedad, materia seca, proteína cruda, fibra cruda, 
extracto etéreo y ceniza de la pastura cultivada disponible en el potrero se realizó de acuerdo con los protocolos de la AOAC (1995).

\section{Composición Botánica del Pasto Consu- mido}

El muestreo se realizó en horas de la mañana, luego de 30 minutos de iniciado el pastoreo. Se identificaron a cinco alpacas y cinco llamas destetadas y se simuló su consumo en 50 estaciones de pastoreo (NRC, 1962). El manejo de las muestras fue similar al utilizado para la determinación de la composición botánica del potrero.

\section{Determinación del Índice de Selectivi- dad (IS)}

Se determinó mediante la fórmula diseñada por Ngwa et al. (2000): IS = \% consumido / \% disponible, donde el $\%$ consumido es el porcentaje de la especie consumida por el animal y el \% disponible es el porcentaje de la especie que está disponible para su consumo en el potrero.

La interpretación del IS fue según Pezo y Skarpe (2009), donde IS $>1.3$ indica que la especie está siendo preferida por sobre otras; IS entre 0.7 y 1.3 indica que la especie es neutra; es decir, que la proporción consumida de la especie y su disponibilidad en la pastura es la misma; IS $<0.7$ indica que la especie es rechazada.

\section{Nitrógeno Ureico Sanguíneo}

La obtención de la muestra de sangre se realizó por punción a la vena yugular, empleándose tubos vacutainer de $3 \mathrm{ml}$ al vacío sin anticoagulante. Las muestras fueron llevadas al laboratorio de la estación experimental para ser centrifugadas a $1372 \mathrm{~g}$ durante $10 \mathrm{~min}$. El suero se almacenó a $-20^{\circ} \mathrm{C}$ hasta su envío al LBNAA-FMV-UNMSM para su respectivo análisis. El tiempo entre la última toma de muestra sanguínea y el centrifugado fue como mínimo de 30 minutos.
La concentración de NUS se hizo mediante el método enzimático, utilizando un kit comercial para la determinación de urea en fluidos biológicos, el cual se encuentra validado para diversas especies, siguiendo el protocolo establecido por Wiener Lab (2000). Las muestras fueron analizadas por triplicado.

\section{Análisis de los Datos}

Los valores de NUS fueron sometidos a la prueba de normalidad de Shapiro-Wilk obteniéndose un valor de $\mathrm{p}$ mayor de 0.05 indicando que los datos siguen una distribución normal. Los datos fueron analizados a través de la prueba t de Student de dos muestras de iguales varianzas.

\section{Resultados y Discusión}

\section{Composición Química del Pasto}

En el Cuadro 1 se presenta la composición química del pasto cultivado ofrecido a los animales del estudio. La composición química de los forrajes se ven influenciados por diversos factores, como la variedad, edad de corte, época de corte, composición florística, riego, etc. Los nutrientes que más se ven afectados son la proteína cruda y la fibra cruda. En el presente estudio, el nivel de proteína cruda fue superior al reportado por Bojórquez (1998) para los periodos de des-

Cuadro 1. Composición química de la asociación rye grass italiano (Lolium multiflorum) y trébol blanco (Trifolium repens) ofrecido a alpacas y llamas destetadas en Cusco, Perú

\begin{tabular}{lc}
\hline Nutriente & $\%$ \\
\hline Materia seca & 18.09 \\
Proteína cruda & 16.30 \\
Fibra cruda & 19.20 \\
Extracto etéreo & 1.43 \\
Ceniza & 6.60 \\
\hline
\end{tabular}


Cuadro 2. Composición botánica del área de pastoreo e índice de selectividad del consumo de alpacas y llamas destetadas

\begin{tabular}{lcccc}
\hline & \multicolumn{2}{c}{ Composición botánica } & \multicolumn{2}{c}{ Índice de selectividad (IS) } \\
\cline { 2 - 5 } & Gramínea (\%) & $\begin{array}{c}\text { Leguminosa } \\
(\%)\end{array}$ & Gramínea & Leguminosa \\
\hline Potrero & 82.1 & 17.9 & & \\
Consumo de alpacas & 78.5 & 21.5 & 0.96 & 1.20 \\
Consumo de llamas & 84.0 & 16.0 & 1.02 & 0.89 \\
\hline
\end{tabular}

Cuadro 3. Nitrógeno ureico sanguíneo $(\mathrm{mg} / \mathrm{dl})$ en alpacas y llamas destetadas alimentadas al pastroreo sobre una asociación rye grass italiano (Lolium multiflorum) y trébol blanco (Trifolium repens) en Cusco, Perú

\begin{tabular}{lcccc}
\hline & Animales (n) & Promedio & $\begin{array}{c}\text { Desvío } \\
\text { estándar }\end{array}$ & Rango \\
\hline Alpacas & 63 & $19.04^{\mathrm{a}}$ & 3.24 & $12.13-26.36$ \\
Llamas & 64 & $19.05^{\mathrm{a}}$ & 3.93 & $8.80-25.69$ \\
\hline
\end{tabular}

Superíndices similares indican promedios estadísticamente similares

canso de 30, 40 y 50 días, mientras que para fibra cruda fue superior para el periodo de descanso de 30 y 40 días.

En estas pasturas, a mayor tiempo de descanso se incrementa el contenido de fibra cruda y disminuye el porcentaje de proteína cruda. En este estudio la pastura tuvo un descanso de 50 días aproximadamente, se realizó durante la época seca (septiembre - octubre) y recibió un riego por inundación cada 10 días durante ese periodo; además, la proporción de leguminosas fue mayor a lo reportado por Bojórquez (1998) y, por lo tanto, de mayor contenido de proteína cruda, ya que estas plantas pueden proveer al suelo el nitrógeno necesario para su crecimiento (Grijalva et al., 1995).

\section{Índice de Selectividad}

La composición botánica del potrero y la composición botánica del consumo se muestra en el Cuadro 2. El índice de selectividad indica que tanto las alpacas y llamas no manifiestan selectividad en su consumo; es decir, que el consumo de gramíneas y leguminosas fue por igual a la composición botánica disponible en la pastura. Este resultado difiere del estudio de San Martín (1996a), quién determinó en las llamas una mayor preferencia por las gramíneas y en las alpacas una mayor selectividad por plantas herbáceas. Esto pudo deberse que el estudio de San Martín fue sobre pastos naturales donde se tenía una amplia variedad de especies para seleccionar. 


\section{Nitrógeno Ureico Sanguíneo}

No se encontró diferencia significativa en los valores de NUS entre alpacas y llamas (Cuadro 3). Davies et al. (2006) igualmente señalan ausencia de diferencia significativa de niveles de NUS entre alpacas y llamas adultas alimentadas con diferente cantidad de proteína cruda ( 9 y $12 \%$ ). Esto puede deberse, que en alimentos de alta calidad proteica, su eficiencia digestiva para tener una mayor utilización del nitrógeno es mínima en ambas especies (Rúa et al., 2017).

Los promedios obtenidos de NUS en las alpacas $(19.04 \mathrm{mg} / \mathrm{dl})$ y las llamas $(19.05 \mathrm{mg} / \mathrm{dl})$ se encuentran dentro de los rangos reportados por Fowler (2010) de 12-28 mg/dl en animales menores de 1 año y por Flores et al. (2015) de 9.33 - $32.67 \mathrm{mg} / \mathrm{dl}$. Sin embargo, los valores se encuentran por debajo de los valores reportados por Bustinza (2001) de 22$46 \mathrm{mg} / \mathrm{dl}$ y por otros investigadores (Davies et al., 2006; Siguas et al., 2007; Pari, 2015; Barreda, 2017), así como superiores a los obtenidos en otros estudios (Burton et al., 2002; Robinson et al., 2004; Rodrigo, 2015); diferencias que podrían deberse al estado nutricional y fisiológico de los animales (Davies et al., 2006).

Barreda (2017) trabajó con alpacas adultas, en tanto que Rodrigo (2015) trabajó con crías. Se debe considerar que las necesidades nutritivas para mantenimiento de los animales adultos son menores que en animales en etapa de crecimiento (Van Saun, 2006) $\mathrm{y}$, por lo tanto, el reciclaje de urea de un animal adulto será menor que en animales de crecimiento, aumentando así los niveles de NUS. En el caso de las crías, el tracto digestivo no está completamente desarrollado, estando reducida su actividad microbiana hasta las 16 semanas de edad (Samaniego, 1977) y, por ello, el uso del nitrógeno va a ser diferente que en animales destetados cuyo tracto digestivo ya está más desarrollado.
Los aspectos anatómicos y fisiológicos de llamas y alpacas son muy similares y si a esto se suma que el consumo de una pastura cultivada de alta calidad proteica $(16.3 \%$ de proteína cruda) presenta un mecanismo de regulación donde el tiempo de retención del alimento y la capacidad de reciclaje de urea en ambas especies disminuye, se puede entender que no hubiera diferencias significativas para el NUS. Las mayores diferencias en estas especies se han observado a favor de las llamas en condiciones de bajo contenido de proteína cruda y alto contenido de fibra en los alimentos donde el tiempo de retención del alimento y reciclaje de urea aumenta (San Martín y Bryant, 1987; Kiani et al., 2015).

Esta similitud entre ambas especies puede estar relacionada con factores nutricionales como disponibilidad de proteína, ingestión de proteína y solubilidad de los componentes proteicos de la dieta (Olazábal et al., 2009), viéndose reflejada en este estudio por su baja selectividad de las especies del área de pastoreo (Ortega et al., 1997).

\section{Conclusiones}

- No existe diferencia en los niveles de nitrógeno ureico sanguíneo entre alpacas y llamas destetadas alimentadas al pastoreo sobre una asociación de rye grass italiano (Lolium multiflorum) y trébol blanco (Trifolium repens).

- No hubo selectividad entre las especies de pastos cultivados por las alpacas y llamas destetadas.

\section{Literatura Citada}

1. [AOAC] Association of Official Analytical Chemists. 1995. Official methods of analysis. $16^{\text {th }} \mathrm{ed}$. Arlington, USA: AOAC. 
2. Arias J, Nesti de Alonso A. 1999. Importancia de los niveles de nitrógeno ureico en leche y sangre en el ganado lechero. Rev Fac Agron Luz 16: 553-561.

3. Barreda JL. 2017. Efecto de la suplementación alimenticia en la fertilidad de alpacas machos y hembras por inseminación artificial. Tesis de Médico Veterinario. Puno, Perú: Univ. Nacional del Altiplano. $50 \mathrm{p}$.

4. Bojórquez C. 1998. Producción de pastos cultivados en tres zonas agroecológicas de la sierra central. Rev Invest Pec 9(1). [Internet]. Disponible en: http://sisbib.unmsm.edu.pe/bvrevistas/veterinaria/v09_n1/produccionp.htm

5. Burton S, Robinson TF, Roeder NP, Johnston NP, Latorre EV, Reyes SB, Schaajle B. 2002. Body condition and blood metabolite characterization of alpaca (Lama pacos) three months prepartum and offspring three months postpartum. USA. Small Ruminant Res 48: 69-76. doi: 10.1016/S0921$4488(02) 00257-2$

6. Bustinza V. 2001. La alpaca: crianza, manejo y mejoramiento. Tomo II. Puno, Perú: Univ. Nacional del Altiplano. 343 p.

7. Davies HL, Robinson TF, Roeder BL, Sharp ME, Johnston NP, Christensen AC, Schaalje GB. 2006. Digestibility, nitrogen balance, and blood metabolites in llama (Lama glama) and alpaca (Lama pacos) fed barley or barley alfalfa diets. Small Ruminant Res 73: 1-7. doi: 10.1016/j.smallrumres.2006.10.006

8. Flores S, Li O, Gavidia C, Hoyos L, Barrios M. 2015. Determinación del perfíl bioquímico sanguíneo hepático y renal en alpacas (Vicugna pacos) aparentemente normales. Rev Inv Vet Peru 27: 196-203. doi: 10.15381/rivep.v27i1.11445

9. Fowler M. 2010. Medicine and surgery of camelids. $3^{\text {rd }}$ ed. Iowa State, USA: Wiley-Blackwell. $644 \mathrm{p}$.

10. García W, Pezo D, Franco E, San Martín F, Novoa C. 1999. Crecimiento post destete y obtención de peso apropiado para el empadre en alpacas y lla- mas. Rev Inv Vet Peru 10: 39-42. doi: 10.15381/rivep.v10i2.6714

11. Grijalva J, Espinosa F, Hidalgo M. 1995. Producción y utilización de pastizales en la región interandina del ecuador. Quito, Ecuador: INIAP. 55 p.

12. Kiani A, Alstrup L, Nielsen MO. 2015. Differential metabolic and endocrine adaptations in llamas, sheep, and goats fed high- and low-protein grass-based diets. Domest Anim Endocrin 53: 9-16. doi: 10.1016/j.domaniend.2015.03.006

13. Ngwa AT, Pone DK, Mafeni JM. 2000. Feed selection and dietary preferences of forage by small ruminants grazing natural pastures in the Sahelian zone of Cameroon. Anim Feed Sci Tech 88: 253 266. doi: 10.1016/S0377-8401(00)00215-7

14. [NRC] National Research Council. 1962. Basic problems and techniques in ranges research. Washington, DC: National Academic Press. p 70.

15. Olazábal J, San Martín F, Ara M, Franco F. 2009. Crecimiento compensatorio de alpacas: efecto de diferentes niveles de restricción energética y proteica. Rev Inv Vet Perú 20: 171-177. doi: 10.15381/rivep.v20i2.603

16. Ortega HH, Arthaus R, Tahoada, Gallardo M. 1997. Niveles de urea y amonio en sangre y leche de bovinos y su influencia sobre la reproducción. FAVE 10: 33-39. doi: 10.14409/ fave.v10i1/2.3042

17. Pari J. 2015. Efecto de la castración en alpacas sobre el metabolismo de compuestos nitrogenados proteicos y no proteicos. Tesis de Médico Veterinario y Zootecnista. Puno, Perú: Univ. Nacional del Altiplano. $55 \mathrm{p}$.

18. Pezo D, Skarpe C. 2009. ¿Cómo determinar las especies forrajeras que prefieren los animales en una pastura con composición florística compleja? Agroforestería en las Américas 47: 85-93.

19. Robinson TF, Roeder BL, Schaalje $G B$, Hammer JD, Burton $S$, Christensen M. 2004. Nitrogen balance and blood metabolites of alpaca (Lama-pacos) fed three forages of 
different protein content. Small Ruminant Res 58: 123-133. doi: 10.1016/ j.smallrumres.2004.09.005

20. Rodrigo J. 2015. Niveles de nitrógeno ureico en sangre y leche de alpacas madre y crías. Tesis de Médico Veterinario. Puno, Perú: Univ. Nacional del Altiplano. $51 \mathrm{p}$.

21. Rúa M, Olazábal J, San Martín H. 2017. Purine derivatives excretion function in relation to the fiber/protein ratio in the diet of alpacas (Vicugna pacos). Rev Inv Vet Perú 28: 62-70. doi: 10.15381/rivep.v28i1.12936

22. Samaniego LG 1977. Estudio morfo fisiológico en el desarrollo postnatal del estómago de la alpaca (Lama pacos). Tesis de Médico Veterinario. Lima, Perú: Univ. Nacional Mayor de San Marcos. $46 \mathrm{p}$.

23. San Martín F. 1996a. Nutrición en alpacas y llamas. Fondo Contravalor Perú-Suiza, CISA/IVITA, Univ. Nacional Mayor de San Marcos. Pub. Cient. IVITA $\mathrm{N}^{\circ}$ 27: $\mathrm{p}$ 3-21.

24. San Martín F. 1996b. Nutrición de camélidos sudamericanos y su relación- con la reproducción. Rev Argentina Produc Anim 16: 305-312.

25. San Martín F, Bryant FC. 1987. Nutrición de los camélidos sudamericanos: estado de nuestro conocimiento. Instituto Veterinario de Investigaciones Tropicales y de Altura. Programa Colaborativo de Apoyo a la Investigación en Rumiantes Menores. Lima, Perú. 67 p.

26. Siguas O, Páucar R, Olazábal J, San Martín F, Vélez V. 2007. Valores bioquímicos sanguíneos en alpacas en dos épocas del año en condiciones de Huancavelica: aportes al perfil metabólico de la especie. En: XX Reunión de la Asociación Latinoamericana de Producción Animal (ALPA). Cuzco, Perú.

27. Van Saun RJ. 2006. Nutrient requirements of South American camelids: a factorial approach. Small Ruminant Res 61: 165-186. doi: 10.1016/ j.smallrumres.2005.07.006

28. Wiener Lab. 2000. Uremia para la determinación de urea en suero, plasma y orina. Argentina: Wiener Laboratorios. 